\title{
“Japan LIVE Dashboard” for COVID-19: A Scalable Solution to Monitor Real-Time and Regional-Level Epidemic Case Data
}

\author{
Wei SU a, Wei FU a, Kojuro KATO ${ }^{\text {b }}$, and Zoie SY WONG ${ }^{\text {c,1 }}$ \\ ${ }^{a}$ Yahoo Japan Corporation \\ ${ }^{\mathrm{b}}$ Faculty of Economics, Keio University \\ ' Graduate School of Public Health, St. Luke's International University
}

\begin{abstract}
Under pandemic conditions, it is important to communicate local infection risks to better enable the general population to adjust their behaviors accordingly. In Japan, our team operates a popular non-government and not-forprofit dashboard project - "Japan LIVE Dashboard" - which allows the public to easily grasp the evolution of the pandemic on the internet. We presented the Dashboard design concept with a generic framework integrating socio-technical theories, disease epidemiology and related contexts, and evidence-based approaches. Through synthesizing multiple types of reliable and real-time local data sources from all prefectures across the country, the Dashboard allows the public access to user-friendly and intuitive disease visualization in real time and has gained an extensive online followership. To date, it has attracted c.30 million visits $(98 \%$ domestic access) testifying to the reputation it has acquired as a user-friendly portal for understanding the progression of the pandemic. Designed as an open-source solution, the Dashboard can also be adopted by other countries as well as made applicable for other emerging outbreaks in the future. Furthermore, the conceptual design framework may prove applicable into other ehealth scaled for global pandemics.
\end{abstract}

Keywords. Emergency response, pandemic, dashboard, digital communication, sociotechnical approach

\section{Introduction}

Digital communication has opened new frontiers in the field of pandemic management. Fagherazzi et al., 2020 [4] have addressed the critical roles and transformative effect of digital health initiatives purposed for COVID-19. One of the most novel initiatives since the appearance of the COVID-19 pandemic is interactive dashboards which are aimed at tracking real time case reporting to the public. Examples of global interactive dashboards dedicated to COVID-19 include the World Health Organization (WHO) Coronavirus Dashboard [10], the Johns Hopkins University Dashboard (recorded $>3$ billion page views) [3], and Our World in Data's COVID-19 Statistics and Research. The extremely high total of web hits is testament to how much public attention these dashboards have attracted and their significance as a pandemic risk communication channel for the general

\footnotetext{
${ }^{1}$ Corresponding Author, Zoie SY WONG, Graduate School of Public Health, St. Luke's International University, OMURA Susumu \& Mieko Memorial St. Luke's Center for Clinical Academia, 5/F 3-6-2 Tsukiji, Chuo-ku, Tokyo 104-0045 Japan; E-mail: zoiesywong@gmail.com.
} 
population. However, the examples above are not designed for live updates at a subnational (i.e. regional) level.

As of Sept 2021, Japan has declared five major states of emergency and about $50 \%$ of the Japanese population were fully vaccinated. The hosting of the 2021 (2020) Summer Olympics and the increased circulation of new SARS-CoV-2 variants (including the Alpha and Delta strains) posed great challenges for local disease management, healthcare service operation, and socioeconomic stability in Japan. Yet no curfew has been enforced and no travel restrictions have been put in place for domestic travel throughout the pandemic. As such, local interventions rely heavily on the population's voluntary response [6] and this necessitates a credible and reliable channel of communication to enable the general population to understand the latest situation of COVID-19 in their country. In this paper we document our practical experience in conceptualizing, designing, and operating a real-time and regional-level COVID-19 epidemic monitoring platform - "Japan LIVE Dashboard" for COVID-19. Through addressing the sociotechnical and context sensitive aspects of the Dashboard design, this paper will also be useful for researchers in understanding the role of informatics in the global pandemics.

\section{Concepts, Design and Methods}

When designing the Dashboard, we critically reviewed existing theories, methods, and practices in relation to user experience (UX) design, socio-technical approaches and disease analytics as applied to digital platforms (in consideration of the specificity of COVID-19 disease communication context). The conceptual design of the platform is portrayed in Figure 1. To investigate the role of digital communication in the face of pandemics, we paid close attention towards a system-level infrastructure purposed for the designated epidemic case communication at an individual-level.

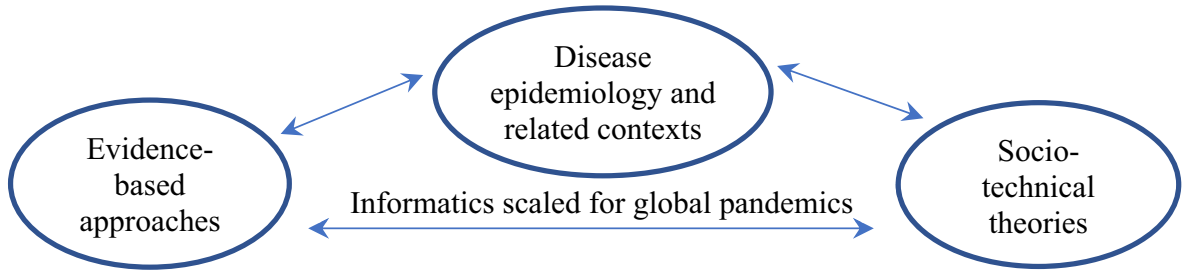

Figure 1. Design concept.

- Socio-technical theories. Pandemic informatics tools are designed to allow users from diverse backgrounds and with different needs to achieve the same task - understanding the latest pandemic status. Recognizing the essential human-computer interactions in the application context, social-technical approaches [1] emphasising the interrelationships between technology and its social environment were adopted.

- Disease epidemiology and related contexts. Informatics scaled for global pandemics should be context-sensitive [9], therefore, disease-related characteristics, transmission dynamics, and trustable up-to-date information sources are essential when building informatics for pandemics.

- Evidence-based approaches. An evidence-based approach [7] has never been more important when dealing with matters of life and death. By drawing upon 
the best available science and evidence-based methodologies, one can work towards informatics scaled for global pandemics.

Apart from the above concepts, in the design phase, special emphasis was placed on high-quality data collection, effective visualization and efficient communication. Several key design features tailored to communicate the current pandemic situation include: (i) a dynamic and interactive dashboard that can visualize case data and statistics from regional areas in real time; (ii) data collection with spatial and temporal scales that potentially allow linked-data analysis among datasets; (iii) a multi-language design; and (iv) an adaptive open-source framework that can be applied to other countries or redeployed to track future epidemics. R Shiny and multiple data visualization and analytics packages, such as "incidence", "EpiEstim", "echarts4r", were incorporated to develop the Dashboard. The automatic deployment workflow is elaborated, as follows. Website data update script operates every 15 minutes to monitor the live data sources using a serverless computing service offered by Amazon Web Services (AWS) Lambda. When there are any data updates, an automatic "Pull Request" will then deliver to our designated GitHub repository, generate the correct format for data visualization, and merge new live data into the main data frame via GitHub Action. Subsequently, CircleCI pipeline will be triggered to update the application on the web server.

\section{Results}

Figure 2 shows the graphical user interface of the Dashboard (http:/COVID2019.live/en/). It was rolled-out on 31 Jan 2020 in Japan, with a speed that was unprecedented at such an early stage of the pandemic. At the time this paper was published, the Dashboard has registered more than 30 million views globally, with $98 \%$ of recorded accesses from domestic users. At the peak of the pandemic, the number of views reached 140,000 per day. This established infrastructure will continue to serve as a major COVID-19 communication tool in Japan until the end of this pandemic. The source code of the platform can be found at the following link (https://github.com/swsoyee/2019-ncov-japan).

Socio-technical aspect. Both social and technology aspects were considered interdependently to enable an effective human-computer interactive design. On the social front, we assessed wide Japanese online users' expectations on tracking updated COVID19 information and aimed to report both country-level and prefecture/regional-level visualizations, statistics and trend projections. All these can be presented under variable epidemic time scales. In addition to the Japanese language, options in English and Chinese further add to its accessibility and community uptake. On the technical front, it is imperative to incorporate intuitive visualizations to effectively communicate complicated disease data with a wide spectrum of online users. Therefore, simple statistics (such as moving average and incidence rate per 100,000 population), choropleth maps, histograms, and infection heat maps were employed.

Disease epidemiology and related contexts. Meaningful COVID-19 disease characteristics and its associated issues that drew public concerns were incorporated into the design. We curated case data on infections, PCR tests, recoveries, deaths, and vaccinations from the real time COVID-19 communication media (including News Digest Japan, the Japanese government (at the national and regional levels)). Since its 
first introduction, this interactive Dashboard has gone through five major iterations to respond to the exigencies of the pandemic at any given time. For future pandemic applications and reproducibility, the versions of these iterations have been documented, and these can be modularized and re-deployed flexibly to other national contexts at different stages of future epidemic outbreaks.

Evidence-based approach. Epidemic theory and dynamic data management were taken into consideration. The epidemic curve illustrates the distribution of the onset of new cases in a graphical format, whereas the time-varying reproduction number [2] is computed to indicate the effectiveness of control measures. Furthermore, we observed that the available case data, reporting structures and formats of these online sources have shifted rapidly as the pandemic situation has evolved. As a result, an automatic and semiautomatic approach for data curation was utilized throughout the course of the pandemic. Automatic web crawling and cross-checking programs were designed to search the latest updates from the designated sites and ensure data consistency drawn from various sources. In order to maintain information integrity and credibility [8], the Dashboard's site manager manually reviews discrepancies and verifies the data on a daily basis.

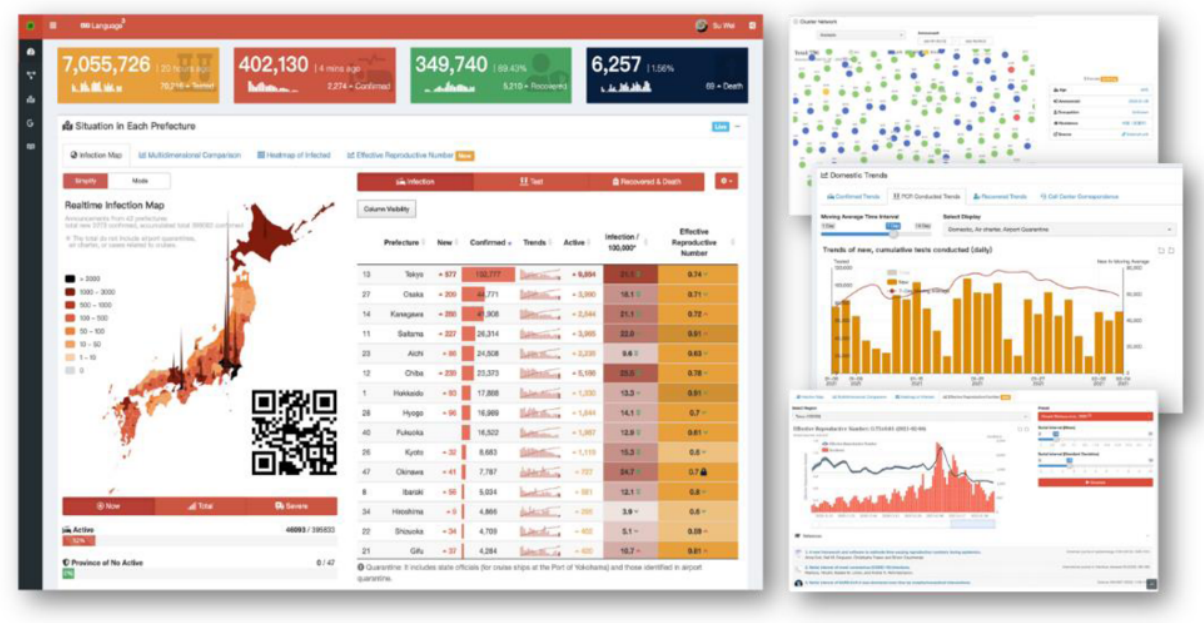

Figure 2. Screenshots of the "Japan LIVE Dashboard" for COVID-19.

We evaluate the readiness of the Dashboard by comparing the case reporting data with the official media and demonstrated a close alignment of reporting accurate case situations in the face of the pandemic. All the data are stored in a machine-readable format, updated in real time and made publicly accessible [5]. The data we collected can serve as a coordinated data repository for ongoing and future disease epidemiology and modeling studies and can thus be potentially used in combination with other disease related datasets with spatial or temporal scales.

\section{Discussion and Conclusion}

Sociotechnical and context-sensitive aspects of digital health were considered in the development of the "Japan LIVE Dashboard" project. Through integrating socio- 
technical theories, disease epidemiology and related contexts, and evidence-based approaches, the digital health conceptual design framework presented proves potentially applicable to other ehealth scaled for global pandemics. Furthermore, the Dashboard provides a scalable solution to visualize local COVID-19 data within a given country's subnational divisions (e.g. states, regions, and even cities) which allows public health authorities, researchers, and the general public to easily understand the evolution of the pandemic. Importantly, the program code for this project is all open source and was written with ease of adaptability in mind. Color, pattern, or shade of all graphical displays in relation to disease variables are adjustable according to user design preference. The digital platform described here is also transferable to other countries and can thus play an important communication role in assisting with future public health emergencies. In the future, this platform could be redeployed to enable investigation of the influence of societal factors on the spread of COVID-19 in Japan. Future study directions include understanding online user preference in obtaining disease information and investigating how to communicate more complicated disease analytics to diverse online population.

\section{Acknowledgements}

This work was supported by the Japan Society for the Promotion of Science KAKENHI grant number $18 \mathrm{H} 03336$.

\section{References}

[1] M. Berg, J. Aarts, and J. van der Lei, ICT in health care: sociotechnical approaches, Methods Inf Med 42 (2003), 297-301.

[2] A. Cori, N.M. Ferguson, C. Fraser, and S. Cauchemez, A New Framework and Software to Estimate TimeVarying Reproduction Numbers During Epidemics, Am J Epidemiol 178 (2013), 1505-1512.

[3] E. Dong, H. Du, and L. Gardner, An interactive web-based dashboard to track COVID-19 in real time, Lancet Infect Dis 20 (2020), 533-534.

[4] G. Fagherazzi, C. Goetzinger, M.A. Rashid, G.A. Aguayo, and L. Huiart, Digital Health Strategies to Fight COVID-19 Worldwide: Challenges, Recommendations, and a Call for Papers, J Med Internet Res 22 (2020), e19284.

[5] L. Gardner, J. Ratcliff, E. Dong, and A. Katz, A need for open public data standards and sharing in light of COVID-19, Lancet Infect Dis (2020).

[6] S. Nomura, D. Yoneoka, S. Shi, Y. Tanoue, T. Kawashima, A. Eguchi, K. Matsuura, K. Makiyama, K. Ejima, T. Taniguchi, H. Sakamoto, H. Kunishima, S. Gilmour, H. Nishiura, and H. Miyata, An assessment of self-reported COVID-19 related symptoms of 227,898 users of a social networking service in Japan: Has the regional risk changed after the declaration of the state of emergency?, The Lancet regional health. Western Pacific 1 (2020), 100011-100011.

[7] M. Rigby, E. Ammenwerth, M.C. Beuscart-Zephir, J. Brender, H. Hypponen, S. Melia, P. Nykanen, J. Talmon, and N. de Keizer, Evidence Based Health Informatics: 10 Years of Efforts to Promote the Principle. Joint Contribution of IMIA WG EVAL and EFMI WG EVAL, Yearb Med Inform 8 (2013), 34-46.

[8] D. The Lancet Infectious, The COVID-19 infodemic, Lancet Infect Dis 20 (2020), 875.

[9] Z.S. Wong, C. Nohr, C.E. Kuziemsky, E. Leung, and F. Chen, Context Sensitive Health Informatics: Delivering 21st Century Healthcare - Building a Quality-and-Efficiency Driven System, Stud Health Technol Inform 241 (2017), 1-5.

[10] World Health Organization, WHO Coronavirus Disease (COVID-19) Dashboard, in, 2020. 